\title{
Intracranial Pressure Monitoring in Traumatic Brain Injury: Start Ventricular or Parenchymal?
}

\author{
Christos Lazaridis ${ }^{*}$ (i)
}

๑ 2019 Springer Science+Business Media, LLC, part of Springer Nature and Neurocritical Care Society

Despite ongoing debate over the merits of intracranial pressure (ICP) monitoring, ICP remains fundamental in the approach to severe traumatic brain injury (TBI). The ICP-based paradigm is endorsed by guidelines and appears to be the dominant way of practicing (in highincome countries at least [1-3]). The question discussed here is as follows: Is the choice of extraventricular drain (EVD) versus an intraparenchymal monitor (IPM) merely a matter of certain patient features in combination with preferences, and convenience of an operator? Or the device may independently contribute to patient outcomes, and consequently, choice ought to be informed accordingly? I assume that most of us would not consider device choice as an independent factor. Bales et al. [4] shared that assumption; however, their analyses proved them wrong. Before delving in, take stock of recent survey data from European centers. One-third of the participants indicated only IPM use, whereas $<10 \%$ indicated that they used only EVDs. In centers that used both, IPMs were used routinely, with drains placed either when the ventricles were enlarged or for cerebrospinal fluid (CSF) diversion [3].

Bales et al. used data from the Citicoline Brain Injury Treatment Trial (COBRIT [5]) to compare severe TBI patients who received an early EVD (within $6 \mathrm{~h}$ of arrival) to those who received an IPM with or without a late EVD. They identified 123 patients with no EVD within $6 \mathrm{~h}$ (IPM group), and 101 patients with an early EVD. A crucial methodological consideration to be understood

\footnotetext{
*Correspondence: Lazaridis@uchicago.edu

Department of Neurology and Section of Neurosurgery, Neurocritical Care, University of Chicago Medical Center, 5841 S. Maryland Ave. MC 2030, Chicago, IL 60637, USA

This comment refers to the article available at https://doi.org/10.1007/ s12028-019-00712-9.
}

before one reads further is "confounding by indication" [6]. When assessing this study, the reader should consider why clinicians select a device, and how decisions might be influenced by factors that also directly affect outcomes. This requires (a) understanding the underlying pathophysiologic mechanisms; in the present case, there is no obvious rationale for why there would be a different impact on outcome, and (b) understanding the criteria for confounding and describing the relationships between potential confounders and both intervention and outcomes. Several characteristics that motivate the selection of ICP-monitoring means could fit as confounders: severity of injury, suspected degree of intracranial hypertension, ventricular size and patency, midline shift, size and location of contusions/hematomas, perceived need for CSF drainage, operator experience, coagulopathy, cost of the monitoring device, and availability, potentially other unknown confounders related to the overall management. Bales et al. went to lengths in order to address confounding. They first used propensity scores; the probability that a patient receives a specific intervention based on their characteristics and the clinical indications determined by the operator. This probability is used to match patients receiving a device with those receiving the comparison to balance potential confounders. Their propensity model included race, Glasgow Coma Scale (GCS) motor, GCS eye plus verbal, number of fixed pupils, and the presence of an epidural hematoma. However, residual confounding may persist due to: (1) "unmeasured confounding," e.g., ventricular size, or coagulopathy; (2) use of a measure for the confounder that does not accurately capture the characteristic it is supposed to represent (e.g., think of the 6-h window and how it may not appropriately classify patients into neatly separated groups of using an EVD as first-line versus later tier intervention for "refractory" ICP); and (3) use of overly broad categories (i.e., even for patients 
with the same value for the variable, there is variability in the likelihood of receiving each device and in experiencing the outcome, e.g., GCS). In a further attempt, the authors specifically compared three sites that almost always used EVDs (47 patients) with two sites that almost never did (77 patients). Further, sensitivity analyses were performed based on timing of EVD placement, as well as comparison of patient outcomes in each group to those predicted from the IMPACT and CRASH models.

All analyses favored the IPM group; $67 \%$ of IPMs had Glasgow Outcome Score-Extended (GOS-E) of 5-8 while only $45 \%$ of the EVD-monitored did, with in-hospital mortality 10\% IPM versus 23\% EVDs. Outcomes were also better at hospitals that primarily used IPMs (although there was no mortality difference). Complications were minimal with either device. There was no significant difference in the daily ICP average or highs. We have no data about intermittent or continuous nor about the reason for CSF drainage. Also, no data provided about "crossover," i.e., what percentage of IPM patients received an EVD for ICP or hydrocephalus management, and how many patients in the EVD group (due to a strategy of continuous drainage) received an IPM. Recently, Volovici et al. have performed a systematic review to compare the effectiveness and complication rate of EVD versus IPM in TBI [7]. Six studies were included with poor overall quality and major risk of bias. The pooled analysis did not show differences in mortality or functional outcomes. The complication rate of EVDs was higher due to infections. Among the included studies, there was one randomized control trial (RCT) by Liu et al. [8], who in a single-center randomized 122 patients, and showed that placement of an EVD was associated with improved ICP control, mortality and GOS at 6 months. The two groups had imbalances with younger patients in the EVD side, slightly different mechanisms of injury, and three times more evacuated hematomas in the IPM patients. Also, their ICP protocol did not mention neuromuscular blockade, temperature control, or barbiturate coma as therapeutic options. Intermittent CSF drainage was followed, and there was no blinding of the outcome assessors.

The safest conclusion is that such a common clinical dilemma deserves to be resolved, and as Bales et al. suggest potentially in a well-defined RCT. Concurrently, we should acknowledge that there are many more such dilemmas, and multichoice debates that are not feasible to address always in an RCT fashion; for this, we need sophisticated comparative effectiveness research methodologies. Until stronger evidence, the current investigation should prompt us toward critical, patient-specific assessment of risks and benefits of each device in terms of operative risk, indication(s) for CSF diversion, place in a tiered approach, method of employment (continuous vs. intermittent), and attention to infectious complications.

\section{Conflict of interest}

The author declare that he have no conflict of interest.

\section{Publisher's Note}

Springer Nature remains neutral with regard to jurisdictional claims in published maps and institutional affiliations.

Published online: 25 April 2019

\section{References}

1. Carney N, Totten AM, O'Reilly C, et al. Guidelines for the management of severe traumatic brain injury. Neurosurgery. 2017;80:6-15.

2. Sivakumar S, Taccone FS, Rehman M, Hinson H, Naval N, Lazaridis C. Hemodynamic and neuro-monitoring for neurocritically ill patients: an international survey of intensivists. J Crit Care. 2017;39:40-7.

3. Cnossen MC, Huijben JA, van der Jagt M, et al. CENTER-TBI investigators Variation in monitoring and treatment policies for intracranial hypertension in traumatic brain injury: a survey in 66 neurotrauma centers participating in the CENTER-TBI study. Crit Care. 2017;21(1):233.

4. Bales JW, Bonow RH, Buckley RT, Barber J, Temkin N, Chesnut RM. Primary external ventricular drainage catheter versus intraparenchymal ICP monitoring: outcome analysis. Neurocrit Care. https://doi.org/10.1007/s1202 8-019-00712-9.

5. Zafonte RD, Bagiella E, Ansel BM, et al. Effect of citicoline on functional and cognitive status among patients with traumatic brain injury: Citicoline Brain Injury Treatment Trial (COBRIT). JAMA. 2012;308(19):1993-2000.

6. Kyriacou DN, Lewis RJ. Confounding by indication in clinical research. JAMA. 2016;316(17):1818-9.

7. Volovici V, Huijben JA, Ercole A, et al. Ventricular drainage catheters versus intracranial parenchymal catheters for intracranial pressure monitoringbased management of traumatic brain injury: a systematic review and meta-analysis. J Neurotrauma. 2019;36(7):988-95.

8. Liu H, Wang W, Cheng F, et al. External ventricular drains versus intraparenchymal intracranial pressure monitors in traumatic brain injury: a prospective observational study. World Neurosurg. 2015;83(5):794-800. 